Chapter 53

\title{
Management of Sepsis
}

\author{
Maurice Rapin and Claude George
}

Recognition of severe sepsis .............................. 885

Factors influencing the choice of treatment................. 887

Treatment.................................................... 892

References............................................ 896

The septic state is defined not only as the occurrence of an infection, but also as the general response of the body towards this infection.

In the critically ill, severe sepsis must be promptly recognized, though this is often difficult to achieve. For this reason the symptoms of severe sepsis are reviewed at the beginning of this chapter.

With the recognition of severe sepsis, three items of information should be sought: the location of infection; the causative organism; and the state of the host's immunity. Only with at least tentative answers to these questions can proper therapy be defined, a point which is developed in the final part of this chapter.

\section{Recognition of severe sepsis}

In the critically ill, recognition of sepsis is often difficult: the common symptoms due to sepsis may be absent whereas others, less commonly encountered, may be prominent. Furthermore, recent results [41] indicate that the clinical picture is not different whatever the microorganism responsible.

\section{Temperature}

Fever is a frequent and early sign of infection and may be preceded by shivering. It is due to the passage of micro-organisms or their toxins into the bloodstream [13]. However, in the critically ill fever may be absent or even replaced by a fall in body temperature [3]. In these circumstances, hypothermia has the same diagnostic and bacteriological implications as a high fever. Furthermore, severe sepsis may be present despite a normal or only marginally raised temperature. This phenomenon is especially frequent in Gram-negative septicaemia and in patients with renal failure or hepatic cirrhosis. The reason for this is unclear but it may be due to a weakened inflammatory response. Since fever in the critically ill may be due to several non-bacterial causes (e.g., deep vein thrombosis, haematomas, drug intolerance), it is necessary to conclude that an abnormality in the temperature chart is a useful alarm signal but does not prove that an infection exists. On the other hand, normal temperature does not rule out severe infection.

\section{Haemodynamic disturbances}

Circulatory shock is frequently the first sign of severe sepsis. Septic shock is easily diagnosed when a low arterial pressure is found, but in fact this is a late sign. Tachycardia, cutaneous and neurological signs, and decreased urine output occur much earlier [14]. The clinical features of septic shock are analysed in Chap. 12.

\section{Acute respiratory distress syndrome (ARDS)}

As discussed previously, ARDS occurs frequently during severe sepsis and may be the 
most prominent feature. This syndrome is associated with non-haemodynamic pulmonary oedema and is usually accompanied by hypoxaemia and hypocapnia related to abnormalities in ventilation: perfusion ratios. Clinically, breathing is rapid and superficial, cough is nonproductive, and murmurs are rare.

Diaphragmatic excursion is decreased, especially with abdominal involvement in the infective process or after laparotomy. Chest $x$-ray examination reveals diffuse and extensive consolidation associated with micro-atelectasias. Pulmonary compliance is decreased, and in patients succumbing to this syndrome intraalveolar hyaline membranes may be found on post-mortem examination. ARDS is an important prognostic factor in severe sepsis [23].

\section{Acute renal failure}

Acute renal failure occurs frequently during severe sepsis. It must be distinguished from simple nitrogen retention due to increased catabolism, in which the blood creatinine is normal. On urine analysis, it is possible to distinguish between functional and organic renal failure: in the former, urine is concentrated, urinary urea concentration is high (usually over 20 times the blood urea), and urinary sodium is low (less than $20 \mathrm{mMol} /$ litre) and less concentrated than urinary potassium. Pathological examination in established renal failure has shown that the lesions involve both renal tubules and the intersitium, although in some cases there is also evidence of a glomerular lesion [5]. The mechanism of acute renal failure during severe sepsis is highly complex and probably several mechanisms can be implicated, including tubular obstruction, an increase in interstitial pressure from oedema, and alterations in renal blood flow associated with a decreased filtration pressure due to dilatation of afferent glomerular arterioles.

\section{Abdominal symptoms and gastric bleeding}

During severe sepsis, whatever its cause, abdominal symptoms are frequent. They usually start with meteorism and reflex ileus and may lead to acute necrotizing enterocolitis. Occasionally, one of the earliest signs of severe sepsis is gastro-intestinal (GI) haemorrhage. Prospective gastric fibroscopy during sepsis has demonstrated that gastro-duodenal lesions occur frequently. They begin as mucosal pur- pura, which is followed by superficial erosions, and the acute ulcers then become haemorrhagic if sepsis is not controlled [25]. Several factors account for these lesions, including mucosal ischaemia, rupture of the mucosal barrier, increased retrodiffusion of $\mathrm{H}^{+}$ions, and reflux of bile salts. They may appear rapidly after the onset of the infection but the bleeding stops abruptly when the infection has been overcome.

\section{Jaundice}

Jaundice occurs frequently during severe sepsis, and is related to hepatic dysfunction due to either infectious hepatitis or the hepatic complications of septic shock [30]. Intrahepatic cholestasis is associated with inflammatory infiltration of the portal tracts compressing biliary canaliculi. Septic shock causes hypoxia of hepatocytes by reducing portal blood flow, whereas hepatic arterial flow is usually preserved. The severity of jaundice is increased by acute renal failure, because excretion of conjugated bilirubin is reduced, and also by increased production of bilirubin consequent upon blood transfusions, resorption of haematomas, or haemolysis induced by bacteria. In the presence of clinically significant jaundice it is essential to exclude biliary tract infection.

\section{Coagulation disorders}

Occasionally in very severe acute sepsis, coagulation disorders are the hallmark of the disease; for example, purpura fulminans is mainly due to meningococcal infections but may also be observed with pneumococci in asplenic patients or even in very severe septicaemias due to Gram-negative bacilli. Sometimes, in vitro study of coagulation indicates the presence of true intravascular coagulation (IVC) thrombocytopenia associated with hypofibrinaemia, and a decrease in factor $\mathrm{V}$ and other co-factors. Experimentally, infusion of endotoxins has been shown to induce IVC in several species by an action on platelets, vascular endothelium, and the coagulation process. In man, however, intravascular thrombi are found far less frequently and IVC may be regarded as incidental [27]. More frequently, fibrin levels are increased and a decrease in partial thromboplastin time related to hepatic failure is observed, in which case the basic trouble is a thrombocytopenia, which may be profound (less than $30000 / \mathrm{mm}^{3}$ ), causing bleeding especially after surgical procedures. Recently the mechanisms of this thrombocy- 
topenia have been studied and increased immunoglobulin $G$ associated with platelets has been found implicating the immune process [21].

\section{Metabolic disturbances}

During severe sepsis several metabolic disturbances occur which are characterized by low recovery of energy metabolism in spite of an increased demand [36]. Hyperglycaemia is due to secretion of cortisol, growth hormone, and glucagon, which increases hepatic gluconeogenesis from amino acids. Hypersecretion of catecholamines causes glycogenolysis and inhibits insulin secretion. Nitrogen catabolism results from increased gluconeogenesis, which causes degradation of numerous important proteins, including those necessary for plasma transport, hepatic enzymes, and those involved in the immune response. Glucose becomes the main supply of energy, thereby increasing insulin secretion which may block fatty acid mobilization.

\section{Encephalopathy}

In the severely ill patient, consciousness is usually depressed as a result of diminished cerebral blood flow. In some patients, however this alteration may be pronounced and of long duration. Examination may reveal myoclonia and asterixis.

\section{The white cell count}

With most types of infection the white cell count (WCC) is elevated, especially when the suppurative process is long-lasting. However, acute sepsis may be associated with the phenomenon of margination of leucocytes and the leucocyte count is consequently low. Similarly, in patients with depressed marrow function, as in folate deficiency, the WCC is reduced. As a consequence, a normal WCC cannot rule out severe sepsis.

\section{Factors influencing the choice of treatment}

\section{Causative organisms}

The identification of the micro-organisms responsible for sepsis depends upon the conven- tional procedures for the isolation of bacteria, viruses, and protozoa. In the vast majority of cases, especially in countries where intensive care is well developed, severe sepsis is related to bacterial infections, and this section is devoted primarily to these.

Knowledge of the site of infection enables a list of 'possible' causative organisms to be compiled. Therefore, specific bacteriological problems are considered for each site of infection.

In theory, bacteriological examination indicates the causative micro-organisms but in practice, except for direct examination of some biological fluids, the examination may take several days, and in some instances no bacteria grow. Whenever possible, all samples for bacteriological study must be taken before antibiotic therapy is started. All results have to be analysed critically; in some situations positive growth may represent contaminants.

\section{Blood cultures}

These should always be performed for it is of the utmost importance to know whether septicaemia is really present, and bacteria isolated from several blood cultures have far greater significance than bacteria isolated from 'local' specimens.

A single blood culture only should never be performed, because of the risk of introducing contaminants from the patient's skin [34]. This is why only one positive culture of a Gramnegative bacterium is of doubtful significance, or conversely why several positive blood cultures are needed to incriminate Staphylococcus epidermidis.

\section{Other examinations}

Colonization of the patient by Gram-negative bacteria after admission to hospital explains why local examinations may have little value, especially sputum, urine, or wound swab examination. The specific procedures for bacterial examination will be emphasized for each location of infection.

\section{Location of infection}

When blood cultures are performed the focus of infection is searched for methodically.

The investigation is based upon the symptoms which have preceded the occurrence of severe sepsis, certain physical signs which may have a localizing value, and complementary 
examinations, especially $\mathrm{x}$-ray and local bacteriological examinations.

Thereafter, surgical treatment of the septic foci should be considered in every case once it has localized.

\section{Lungs and pleura}

There are several types of agent that can cause pleuropulmonary infections, but with bacterial (especially anaerobes) and less frequently viral agents the clinical picture may be one of severe sepsis, sometimes dominating the symptoms of acute respiratory failure (ARF).

Clinical features. These are many, and include coryza, sore throat, cough with purulent, rustcoloured, or bloody sputum, pleuritic chest pain, and dyspnoea. The history should be checked for pharyngeal infection, alcoholism, or difficulty in swallowing, and the possibility of pleuropulmonary infection should always be considered after a general anaesthetic has been administered.

Symptoms of severe sepsis are associated with intense cyanosis, flaring of the ala nasi during inspiration, intense dyspnoea with tachypnea, inspiratory rales, and perhaps signs of consolidation and pleural friction rub or evidence of pleural fluid.

Investigations. Usually only an anteroposterior chest x-ray examination is possible at the bedside; it may reveal opacification of the lungs with a lobar or diffuse patchy distribution. Whereas the chest film is seldom diagnostic of a specific organism, it may be suggestive: lung abscesses are frequently multiple in the case of staphylococcal pneumonia, especially in children. Necrotizing pneumonia caused by aspiration of oral anaerobic organisms also causes abscesses.

Bacteriology. When pneumonia is suspected, a bacteriological examination of the sputum should be performed; this is helpful when the sputum is purulent or bloody but not particularly so when the cough is non-productive, when any isolates should be considered with caution. Very often in hospitalized patients growth of Gram-negative bacteria in the sputum is the result of oropharyngeal contamination. Direct aspiration of bronchial secretions may be more helpful in difficult cases, and samples can be obtained with transtracheal aspiration, or via an endotracheal tube or fiberoptic bronchoscope, although the last two techniques can introduce pharyngeal flora into the bronchial tree. Aspiration of a pleural effusion, if present, may also yield valuable bacteriological information.

The diagnosis of viral infections is made by isolation of the virus in the sputum and by serologic assays. Mycotic or parasitic organisms infect predominantly immunosuppressed patients.

Surgery. Drainage of lung abscesses is helped by bronchial aspiration, physiotherapy, and an inclined position. Purulent pleural effusions should be evacuated as completely as possible by aspiration or surgical drainage.

\section{Peritoneum}

The clinical picture of an intraperitoneal infection varies according to whether the infection is initially generalized (peritonitis) or localized (intraperitoneal abscess).

Peritonitis. In secondary bacterial peritonitis, the numerous species of both aerobic and anaerobic micro-organisms that occur in the GI tract may all be found. In contrast, primary bacterial peritonitis is usually seen in patients with ascites and is often due to streptococci and Enterobacteriaceae.

Clinical features. These include abdominal pain, fever, and chills; abdominal distension, diffuse and/or localized tenderness with rigidity of the abdominal wall and absent bowel sounds are characteristic. The location of the pain and tenderness may suggest the nature of the underlying cause. Findings are usually less prominent at both extremes of age, in alcoholics, and in patients with ascites or receiving steroids.

Investigations. Supine and upright straight $\mathrm{x}$-ray examination of the abdomen may show air in the peritoneal cavity, evidence of ileus or peritoneal fluid. In doubtful cases, peritoneal puncture or catheterization may be helpful in the diagnosis.

Bacteriology and surgery. After diagnosis, prompt surgery is indicated in most cases, the degree of urgency depending on the severity of the sepsis. Except in cases of primary peritonitis, bacteriologic examination of peritoneal pus is of little significance, since it always contains organisms from the GI tract. If no anaerobic strains are found it probably implies incorrect sampling.

Intraperitoneal abcess. After an intraperitoneal infection or any abdominal operation, subphrenic or pelvic abscesses may develop.

Clinical features. The onset of these is usually insidious, with low-grade fever, diarrhoea, abdominal distension, abdominal or thoracic 
pain, and finally the symptoms of severe sepsis. Localized tenderness of the abdomen, basal ileus (in the case of subphrenic abscess), or findings consistent with a pleural effusion may be present.

Investigations. The most consistent findings seen on chest and abdominal $x$-ray pictures are elevation of the diaphragm, basal atelectasis, pleural effusion, and gas-fluid levels. Localization of the abscess may be achieved by ultrasonic or CAT examination, possibly in association with a gallium scan [8]; sinograms may be possible in postoperative patients.

Surgery. After proper localization the abscess should be evacuated, care being taken to avoid seeding of the peritoneal cavity.

\section{Female pelvis}

As a complication of septic abortion or in association with pelvic thrombophlebitis, pelvic infection in the female can be life-threatening because of the presence of anaerobic organisms, particularly clostridial species.

Clinical features. A history of purulent and/or bloody vaginal discharge or of a preceding abortion is usually obtained.

Lower abdominal pain is a feature and serosanguineous material may be present in the vaginal vault. Following an abortion the uterus is enlarged and tender.

Investigations and treatment. After several blood cultures have been taken (cultures of the vaginal or uterine discharge are of no value) and antibiotics effective against anaerobes administered careful curettage of the uterus should be performed, even if most of the products appear to have been expelled spontaneously. If the patient's condition does not improve rapidly a hysterectomy must be considered.

\section{Bile ducts}

Infection of the bile ducts is usually associated with obstruction of the biliary tract.

Clinical features. Acute colicky pain in the right upper quadrant, rigor, and fever followed by jaundice are the typical symptoms. Sometimes the jaundice is more marked than the history of pain.

Examination may reveal right upper quadrant tenderness and guarding. Signs of a pleural effusion and pain on percussion of the liver are suggestive of an associated hepatic abscess, but this is extremely difficult to diagnose and can occur without biliary obstruction, for example in appendicitis, amoebic infection, or staphylococcal septicaemia.

Investigations. An ultrasound scan of the liver is the investigation of choice, or if this is not available abdominal angiography or radioisotope scanning may be used. Liver function tests show an elevation of conjugated bilirubin and moderate increases in alkaline phosphatase and the transaminases.

Surgery. When the infection of the bile ducts is associated with symptoms of severe sepsis immediate surgery is needed to relieve bile duct obstruction; this also makes it possible to perform a bacteriological examination of the bile, which very often grows $E$. coli. In some cases where a stone is at the lower end of the bile duct, endoscopic sphincterotomy may be possible.

\section{Urinary tract}

Numerous factors predispose to urinary tract infection, but the main one is obstruction to urinary flow of any origin. The common causative organisms are E. coli, Proteus, Enterobacter, group D Streptococcus, Klebsiella, and Pseudomonas.

Clinical features. A history of urgency and frequency of micturition, dysuria, haematuria, and nocturia may precede the onset of infection, which in turn will increase the intensity of these symptoms.

In the case of pyelonephritis or an intrarenal or perinephric abscess, pain and tenderness in the costovertebral angle is frequent, and this may be due to renal enlargement.

Investigations. Examination of the urine typically reveals mild proteinuria and haematuria. Quantification of organisms in the specimen discloses a number of organisms in excess of 100000 and the number of white cells is frequently increased.

Urgent intravenous pyelography may identify a predisposing factor, but may not identify an intrarenal or perinephric abscess. Ultrasound and CAT are then the imaging techniques of choice $[1,32]$.

Treatment. When urinary retention or a major abscess is found appropriate surgical relief is mandatory.

\section{Meninges}

Clinical features. In a patient with severe sepsis the diagnosis of meningitis can be relatively easy, with headache, neck stiffness, and Kernig 
and Brudzinski signs all being present. However, the first signs are sometimes an altered level of consciousness and convulsions, without much in the way of localizing signs.

Investigations. In every case, lumbar puncture allows clinical and bacteriological diagnosis. Bacterial meningitis may be suspected when analysis of the cerebrospinal fluid (CSF) shows a low sugar level and a raised polymorphonuclear cell count. The organisms most frequently isolated in primary meningitis are meningococci, pneumococci, Haemophilus influenzae (especially in children), and Listeria; antibiotic treatment must be started immediately. A lymphocytic reaction in the CSF is most frequent in viral or tuberculous meningitis but may be found initialy with Listeria infection.

When focal neurological symptoms are present, encephalitis, thrombophlebitis or a cerebral abscess have to be looked for by means of CAT or other neuroradiological investigations.

\section{Catheter-related}

In patients in ICUs, infections related to intravascular catheters are frequent and serious. The main organisms responsible are Staphylococcus aureus, Gram-negative bacilli, and fungi, especially Candida albicans. Except when another site is clearly responsible for the infection, the catheter should be removed and its tip cultured. In many instances a complete recovery follows without antibiotics, but if the signs persist appropriate antibiotics should be given. Persisting positive blood cultures after removal of the catheter and antibiotics may be indicative of deep venous phlebitis or endocarditis.

\section{Endocarditis}

Cardiac valves may be involved by a wide variety of organisms, although Streptococcus and Staphylococcus are more likely than others to induce extensive damage.

Clinical features. Because of its widely variable clinical presentations, infective endocarditis is difficult to diagnose early, especially in a patient with severe sepsis. The sudden onset of congestive heart failure may be apparently related to septic shock and then a history of valvular heart disease is helpful but not essential.

The finding of a new heart murmur is of great value; however, murmurs may be absent in patients with right-sided endocarditis, which is especially frequent in drug addicts and after IV catheter infections. Finally, endocarditis should always be considered when the infection is disseminated to many organs, such as the CNS, spleen, kidneys, bones and joints. Cutaneous symptoms (Osler nodes, Janeway lesions) should be looked for on the palms of the hands and soles of the feet.

Investigations. Ultrasound is helpful because of its non-invasive nature, but when pulmonary oedema is extensive and cardiac surgery is contemplated, formal cardiac catheterization with angiography is necessary.

\section{Soft tissues}

Some soft tissue infections yield aerobic organisms, and less frequently anaerobic organisms, although there is good evidence to indicate that the latter are initially responsible. Gas gangrene is caused by several species of clostridii; aerobic and anaerobic streptococci are usually responsible for cellulitis.

Clinical features. Within several hours or days of injury or surgery of the GI tract, increased swelling and pain develop, with oozing of serous or serosanguineous material around the particular site.

On palpation the wound is very tender, and crepitations indicative of gas may be felt. Eventually bullae may cover the wound, beneath which the muscle tissue is pale and bleeds very little. If haemolysis occurs the patient rapidly becomes jaundiced and haemoglobinuria may occur.

Treatment. Immediate medical and surgical intervention is necessary. If possible hyperbaric $\mathrm{O}_{2}$ should be given but this should not delay the surgical removal of dead tissue, which may require amputation of an extremity or extensive debridement.

\section{Miscellaneous}

Severe sepsis may be caused by a miscellany of organisms of the following types: bacterial (typhoid fever, leptospirosis), mycobacterial (tuberculosis), protozoal (malaria), and viral. The usual diagnostic procedures should be performed as in less severe cases.

\section{Host defence mechanisms against infection}

The management of the critically ill patient would not be complete without consideration of the state of the host defence mechanisms against infection. Many factors account for the increased susceptibility of critically ill patients to 
infection. Mechanical barriers against bacteria are weakened by instrumentation (endotracheal intubation, IV catheterization, urinary catheterization) and various surgical procedures. Defence mechanisms may be altered by drug therapy (steroids or cytotoxic drugs), surgery, and numerous underlying conditions (cirrhosis, malignancy, trauma, burns, diabetes, and renal failure). Furthermore, severe sepsis itself induces immunological disturbances. Thus the state of the host defence mechanisms against infection may be considered from the aspects of skin testing, cellular and humoral immunity, and the changes found in septic shock.

\section{Skin testing as a means of assessing host defence mechanisms}

Recent studies have indicated that the compromised host, who is highly susceptible to severe infection, may be recognized by delayed hypersensitivity to skin testing (DHST) [11]: in patients suffering from severe acute illness, a failure of the DHST response (anergy) is associated with high mortality, mainly from sepsis. In several studies carried out in ICUs, the mortality rate was $57 \%-58 \%$ in anergic patients, whereas when at least one response (e.g. induration greater than $5 \mathrm{~mm}$ ) was recorded it was $16 \%-18 \%$ [11]. This relationship was initially shown in surgical patients, in whom the presence of anergy before surgery was associated with an increased incidence of postoperative septic complications [32]. The tests consist in intradermal injection of standard recall antigens, the most frequent being tuberculin, Candida, and streptokinase, which may be associated with skin tests with phytohaemagglutinin, dinitroclorobenzene or croton oil.

Old age and disseminated cancer apart, anergy may be due to several factors:

1) The effects of malnutrition have been studied extensively: either protein or calorie deprivation appears to alter the delayed hypersensitivity reactions, a defect which can be corrected by an appropriate diet.

2) It is firmly established that infections such as severe tuberculosis or measles are associated with a reduction of anergy. However, several other bacterial, viral, or parasitic infections have also been observed to produce the same effect [19]. The underlying process is rather complex, but antigenic stimulation and $T$ cell depletion appear to be the most salient features.

3) Following surgery or trauma, both delayed hypersensitivity reactions and other immunological responses are depressed. There are de- creased numbers of circulating lymphocytes and those that form $E$ rosettes; leucocyte chemotaxis is reduced either under basal conditions or after stimulation with caseine: furthermore, immune depressant factors circulate in these patients [10].

\section{Cellular and humoral immunity}

Both circulating bacteria and endotoxin are cleared mainly by polymorphonuclear neutrophils and the hepatic reticuloendothelial system. This explains why serious infections frequently occur when leucocyte production is depressed or in the presence of portocaval anastomosis, as in hepatic cirrhosis. In contrast to the elimination of bacteria by the hepatic reticuloendothelial system, a non-specific phenomenon independent of the antibodies, phagocytic function of polymorphs appears to be dependent on its presence. Weinstein and Young [40] have shown that $40 \%$ of patients with septicaemia are infected by strains of bacteria that are resistant to phagocytosis by polymorphs. They found, however, that this defect was not related to an intrinsic white cell deficiency but to the absence of specific opsonizing antibodies; during recovery the capacity for phagocytosis is restored simultaneously with the rise in antibody titre.

From the time of colonization of the digestive tract by Gram-negative bacteria, antibodies will be formed against the serotypes of the bacteria present. These antibodies, principally of the IgM type, are known as natural antibodies, and in the presence of complement they confer on the serum a bactericidal power active against numerous strains of Gram-negative bacilli. The attachment of activated complement to the bacteria produces a lytic action causing bacterial death.

Thus a failure of the hepatic reticuloendothelial system, neutropenia, the presence of bacteria of an unknown serotype or the lack of opsonizing antibodies are conditions that may be found in the case of Gram-negative septicaemia. Similar factors account for infections due to cocci. Decreased humoral immunity raises the frequency and the severity of infections due to Streptococcus pneumoniae: fulminating infections due to this organism have also been described in asplenic patients.

\section{Immunologic alterations due to septic shock}

Several studies have shown that levels of complement components decrease during septic 
shock [11]. This decrease appears to be related to activation of complement via either classic or alternative pathways, and it has been found during infections due to Gram-negative bacilli, Gram-positive bacteria, and viruses. Serum immunoglobulins are decreased simultaneously and the two factors may be responsible for the major impairment in host defences. The presence of circulating immune complexes has been demonstrated during several infectious diseases, irrespective of the infecting organism. However, they cannot be detected during lethal septic shock [17]. The significance of this finding remains to be elucidated.

\section{Treatment}

\section{Antimicrobial drug therapy}

Several studies have shown that bacteria isolated from patients admitted to critical care units (CCUs) with severe sepsis were much more frequently multiresistant than those isolated from other parts of the hospital [38]. This is one reason why superinfections are more frequent and life-threatening in these patients [37]. The widespread and uncontrolled use of antibiotics has caused these phenomena and illustrates the need for a strict antibiotic policy [16].

\section{Rules for antimicrobial drug therapy in CCU patients}

Monotherapy. As often as possible, antimicrobial chemotherapy should be undertaken with only one drug. The combination of two antibiotics increases the risk of the emergence of multiresistant strains and should be restricted to a limited number of infections, namely tuberculosis, mixed bacterial infection, bacterial endocarditis, and severe infection with Gram-negative rods in immunosuppressed patients.

Narrow-spectrum drug. When two drugs having an equal efficacy are considered, the one with the narrower spectrum should be preferred, because it is less likely to have a deleterious action on commensal bacteria and to increase the proliferation of those which have become resistant.

Restriction of antibiotic prophylaxis. The use of antibiotics enhances the emergence of bacterial resistance to useful antibiotic agents [24]. Therefore, antibiotic prophylaxis should be restricted to clinical situations where controlled and prospective studies have clearly shown its efficacy in preventing the emergence of superinfections $[7,9,20]$. In critical care medicine, the circumstances in which it is most valuable are traumatology and surgery.

After surgical procedures, chemoprophylaxis appears to be beneficial in the case of operations in which endogenous bacterial contamination of the wound is unavoidable. It should also be considered for patients who require the insertion of foreign materials, e.g., prosthetic heart valves. In these cases, a simple dose of one antibiotic should be injected immediately before the start of the operation [39].

There is no definite evidence that prophylactic antibiotic therapy prevents superinfections following instrumental techniques for diagnosis or treatment, such as tracheostomy, bladder catheterization, and intravenous or intra-arterial catheterization.

Adherence to such rules for treatment and prophylaxis has considerably reduced the incidence of septicaemia due to superinfection from more than $10 \%$ to less than $5 \%$ of CCU patients [31].

\section{Choice of antimicrobial drugs in CCU patients}

Antimicrobial agents should be chosen with the following aims in mind:

- The antibiotic must be active against the bacteria directly responsible for the sepsis that one has to treat or prevent.

This means that bacteriological findings made in the laboratory must be critically examined. Providing that meticulous techniques are used, bacteria isolated by blood cultures, lumbar puncture, or bladder catheterization have an unequivocal value. On the other hand, in certain circumstances it is difficult to determine whether the identified strains are really responsible for the infection or result simply from colonization. Such dilemmas occur with the bacterial examination of specimens of sputum, tracheal aspirate, pus, wound dressings, etc. In such circumstances it is often possible to define the type that might be responsible by taking into account the locality of the sepsis and the usual epidemiological considerations, or by means of bacterial examinations that should be more specific, e.g., transtracheal aspiration of secretions.

- The antibiotic used should have the appropriate pharmacological and pharmacokinetic properties to 
allow it to reach the infected focus in a sufficient concentration.

- The frequency and severity of adverse drug reactions should be weighed against the beneficial effects.

- The financial cost should be as low as possible without reduction of benefit.

For these aims to be achieved, the different drugs may be separated into three groups. (Ball and Geddes [2] reviewed some new antibiotics in 1979.)

First group. The combined properties of these antibiotics are a narrow spectrum, a low incidence of adverse reactions, and a low risk of inducing proliferation of multiresistant Gramnegative rods. They should be chosen in every case in which bacteria susceptible to them are responsible for the infection.

Penicillin is the first drug of choice in infections due to streptococci, including S. pneumoniae (Pneumococcus) and anaerobic streptococci (Peptostreptococcus sp.), to Neisseria meningiditis, and to Clostridia.

The most frequent indications for the use of penicillin in critically ill patients are (1) Chemoprophylaxis of gas gangrene and other anaerobic infections in patients with a high risk of clostridial superinfection, i.e., postpartum or post-abortion infections, deep wounds, especially after road traffic accidents, open fractures, colonic and perineal surgery, especially in diabetics, and surgery for peripheral vascular disease; (2) acute pneumonia in patients with previously normal lungs, especially pneumococcal (primary pneumonia) or anaerobic (post-inhalation, foul odour, alcoholic patients with poor dental hygiene); and (3) acute meningitis due to $N$. meningiditis, $S$. pneumoniae, or Listeria monocytogenes needing combined therapy with an aminoglycoside (preferably gentamicin).

Besides allergy, the principal side-effects of penicillin are related to high blood levels which develop in the presence of renal insufficiency; they include metabolic encephalopathy (depressed consciousness, abnormal movements, and convulsions) [29] and bleeding due to functional platelet abnormalities [35]. These adverse effects disappear when the dosage is reduced.

Isoxazolyl-penicillins (methicillin, oxacillin, cloxacillin), which are resistant to beta-lactamases secreted by staphylococci, are the drugs of choice in infections due to staphylococci. Nevertheless, the sensitivity of staphylococci is very different from one strain to another and cannot be estimated from the bacteriostatic action in vitro alone. Therefore, the choice must be con- firmed by, or modified in accordance with, the results of the in vitro bactericidal capacity of the antibiotic against the responsible strain.

When sepsis is severe, especially in septicaemia, combination with an aminoglycoside (gentamicin) is recommended because of its synergistic action with isoxazolyl-penicillins against staphylococci.

Macrolides (erythromycin) are the best alternative to penicillin in cases of streptococcal infections and of allergy to penicillin.

They are also efficient against other organisms, such as Mycoplasma pneumoniae, Chlamydia trachomatis, Legionella pneumophila and many anaerobic bacteria. For this reason, the macrolides are valuable antibiotics, highly recommended for use in acute, apparently primary, pneumonias.

Because of their good tissue penetration, their spectrum, and the low incidence of side-effects, they are the antibiotics of choice for the treatment of superinfections in chronic obstructive airways disease with acute respiratory insufficiency and encephalopathy [26].

Nitroimidazole compounds (metronidazole, ornidazole) are the drugs of choice against obligate anaeorobes, especially Bacteroides fragilis; aerobic and facultatively anaerobic bacteria are naturally resistant. Nitroimidazoles are highly effective in the treatment and prophylaxis of a wide variety of non-clostridial anaerobic infections and are compatible with many other antimicrobial agents [18].

Imperative indications include bacteraemia, endocarditis, meningitis, brain abscess, pneumonia, cellulitis, and necrotizing myositis or fasciitis due to obligate anaerobes. In addition, it is advisable to combine nitroimidazoles with surgical drainage from localized infections, which are known to include a high risk of anaerobic bacteraemia, such as intra-abdominal infections and pelvic sepsis originating from the female genital tract. In these cases, a useful preventative measure against aerobic Gramnegative bacteria is a combination of nitroimidazole compounds with gentamicin. Despite their lack of activity in vitro against aerobic organisms, nitroimidazoles have been shown to be effective both in surgical prophylaxis and in the treatment of mixed aerobic-anaerobic infections: aerobic and anaerobic bacteria disappear as the sepsis resolves in response to nitroimidazoles [15]. Their prophylactic use has been conspicuously successful in the prevention of postoperative sepsis in surgery of the GI and female genital tracts.

Quinolone compounds (nalidixic acid, pipemidic 
acid) are mostly active against urinary infections caused by strains of Escherichia coli and Proteus mirabilis, when the sepsis has not invaded the kidney or related systems such as the prostate and when high tissue concentrations are not necessary. These compounds should be used preferably and initially in urinary infections due to long-standing bladder catheterization, when the infection has not been cured by removing the catheter and increasing the urinary output. Second group. The joint property of this group is a wide spectrum inducing ecologic damage to commensal flora. On the other hand, some of these drugs have significant toxicity. However, these deleterious effects may be overlooked when these drugs are found to be effective against bacterial stains resistant to drugs of the first group.

For this reason, drugs in this group should be selected only when one is sure that drugs in the first group would be inactive.

Aminopenicillins (ampicillin, amoxycillin, metampicillin): the rising incidence of infections due to Gram-negative rods observed in surgical wards and CCUs since 1965 seems to be related to the wide use of aminopenicillins. Furthermore, these drugs are probably the ones most frequently implicated in the development of plasmidic resistance. They are also the antibiotics most frequently associated with pseudomembranous colitis due to Clostridium difficile toxin [4]. Therefore, the use of aminopenicillins should be restricted to those infections in which this group of antibiotics is irreplaceable.

Aminopenicillins are indicated in infections due to many strains of $E$. coli, P. mirabilis and Klebsiella pneumoniae for community-acquired infections. On the other hand, an increasing frequency of resistant hospital strains has been observed. Aminopenicillins have a special place in urinary tract infections due to these microbes, where widespread diffusion of the antibiotic is mandatory, i.e., in pyelonephritis.

The pharmacokinetics of aminopenicillins with their high biliary elimination support their preferential use in biliary tract infections, and IV metampicillin, has the highest biliary concentration.

Aminopenicillins are the most active antibiotics against Haemophilus influenzae. They should be used initially in infections where the bacterium has been isolated (pneumonia, meningitis) or before isolation when infection with $H$. influenzae is a possibility as in bacterial meningitis, especially in children.

Gentamicin remains the most frequently pre- scribed aminoglycoside antibiotic. The nephrotoxicity and ototoxicity of all aminoglycosides makes one cautious about their use in cases of renal insufficiency, or hypovolaemia or in association with the loop diuretics and other antibiotics such as the cephalosporins.

Their tissue distribution is poor, but because of their toxicity this disadvantage cannot be overcome by increasing dosage.

Aminoglycosides are potent against a great number of the bacteria responsible for some of the most serious infections seen in critically ill patients. But they do have a powerful tendency to induce resistant strains.

They are inactive against many strains of streptococci and against anaerobes, and are mainly used in association with compounds of other families when synergism is needed, as in severe sepsis due to staphylococci, Enterobacteriaceae and Pseudomonas Spp. However, in the last two instances, the new cephalosporins are tending to take their place.

Cotrimoxazole is potent against several bacterial species, especially streptococci and some Enterobacteriaceae such as E. coli and P. mirabilis; it has good tissue penetration [33]. In the cases of intolerance or resistance to the aminopenicillins, this drug might be used for serious or recurring infections of the urinary tract, especially in cases of pyelonephritis or prostatis. Its efficacy against Pneumocystis carinii makes it useful in opportunistic infections in immunodepressed patients.

Colistin is the main antibiotic of the polypeptidic group. In vitro, it is active against many strains of Enterobactericeae and Pseudomonas. It has a low tendency to induce plasmidic resistance but unfortunately, its in vivo efficacy is limited by its low diffusion, which cannot be overcome by increasing the dose because of its nephro and neurotoxicity. Its use in critical care medicine is therefore restricted.

Third-group. These drugs share either a high efficacy against several microbial strains or high financial cost. The emergence of bacterial resistance to each new antibiotic introduced into medical use is a phenomenon that has been repeatedly documented. Therefore these highly active new compounds should be reserved for specific cases to retain their efficacy for as long as possible.

They are indicated when in vitro testing shows that no drug of the preceding group is active and they should be reserved for serious life-threatening infections.

Carbenicillin and other semi-synthetic penicillins, such as ticarcillin, mezlocillin, and azlocillin, 
have a broad spectrum of activity which includes 'problem' organisms such as Pseudomonas aeruginosa and the less common Proteus species ( $P$. vulgaris, $P$. morganii and $P$. rettgeri). The potential of these antibiotics is limited by their susceptibility to beta-lactamases and by resistance to R-factors which may be acquired during treatment.

They may be useful in combination with an aminoglycoside in undiagnosed life-threatening infections in neutropenic patients.

Cephalosporins. Recently several new cephalosporins have been introduced. Some of these are highly insensitive to beta-lactamases: cefuroxime, cefamandole, cefoxitin (the first of a new family of antibiotics called cephamycins), cefotaxim, and cefsulodin; the last two are of special interest in critical care medicine.

Cefotaxim is the most potent antibiotic against Enterobacteriaceae and its resistance to inactivation by beta-lactamases is greater than that of other cephalosporins. Its minimum inhibitory concentration is the lowest of all known antibiotics active against Gram-negative bacteria, with the exception of Pseudomonas aeruginosa and obligate anaerobes (Bacteroides fragilis).

Cefotaxim is particularly useful in severe hospital-acquired infections due to multiresistant Gram-negative rods (Escherichia coli, Klebsiella sp., Proteus sp., Serratia sp.), especially during nosocomial bacteraemia and extensive pneumonia. Sufficient concentrations are achieved in CSF, and the successful treatment of meningitis by cefotaxim has been reported [6].

Cefsulodin has a very low minimum inhibitory concentration against Pseudomonas aeruginosa but poor efficacy against Enterobacteriaceae. Thus this compound may be considered as a narrow-spectrum antibiotic. It is now the first antibiotic of choice in serious infections due to Pseudomonas aeruginosa, especially in septicaemias and necrotizing pneumonias.

The new aminoglycosides, tobramycin, netilmicin, and amikacin, should be reserved for the management of serious infections caused by gentamicin-resistant organisms [28].

Extension of acquired resistance against one antibiotic (thus reducing the real spectrum of activity) is related to the duration of its use and therefore the oldest of the active drugs should be chosen.

Vancomycin is a nephrotoxic antibiotic that is highly active against staphylococci. It should be reserved for serious infections caused by that organism [12] and by Clostridium difficile.

Antibiotics of limited use in critical care. The following preparations have no or very few indications:

The first and second-generation cephalosporins (namely cephaloridin, cephalothin, cephalexin, cephacetril, cephanon, cephapirin, cefazolin and cefatricin) all have the disadvantages of the broad-spectrum antibiotics, and their widespread use has been recognized as being responsible for the development of nosocomial Gram-negative sepsis, especially that due to Pseudomonas aeruginosa. It is now possible to choose other drugs with fewer drawbacks in all cases.

Tetracyclines are bacteriostatic, broadspectrum antibiotics, highly implicated in the development of bacterial resistance. The frequency with which resistance is found among streptococci, staphylococci, Enterobacteriaceae, Clostridia, and Bacteroides is so high that tetracyclines can no longer be advocated.

The phenicol antibiotics (chloraphenicol, thiamphenicol) have followed the same evolution as the tetracyclines, and furthermore their bone marrow toxicity makes them hazardous. However, because of the good CSF concentrations, they still have a definite advantage in treating meningitis due to beta-lactamase producing bacteria, and they are still the drugs of choice in enteric fever.

Lincomycin and clindamycin are active against streptococci, staphylococci, and Bacteroides, and their main advantage has been in their activity against Bacteroides. They have been proved to be the commonest cause of antibiotic-associated pseudomembranous colitis however, and there fore, since the introduction of nitroimidazoles there have been few, if any, indications for lincomycin and clindamycin in the prophylaxis or treatment of anaerobic infections.

Several active bactericidal compounds are now available for testing most of the severe infections found in CCUs.

The question that remains is how to adapt a strategy for their most effective use whilst keeping their unavoidable side-effects to a minimum. In this context it is worth remembering that many of the problems in severe sepsis are related more to a depressed host response or to difficulties in eliminating septic foci than to a lack of suitable antibiotics.

\section{Treatment of the infected focus}

Antibiotics are not the only weapon available for treating sepsis, and they are not even always the most effective one. Usually a septic condition is secondary to an infected focus, and the 
Table 53.1. Efficacy of treatment of aerobic Gram-negative rod septicaemia in 77 patients

\begin{tabular}{lll}
\hline $\begin{array}{l}\text { Treatment of the } \\
\text { septic focus }\end{array}$ & Effective & \multicolumn{2}{c}{$\begin{array}{l}\text { Ineffective or } \\
\text { ineradicable }\end{array}$} \\
\cline { 2 - 3 } Antibiotic therapy & $\begin{array}{l}\text { Appropriate } \\
\text { Yes }\end{array}$ No & $\begin{array}{l}\text { Appropriate } \\
\text { Yes }\end{array}$ \\
\hline & & Nortality (\%)
\end{tabular}

discovery and eradication of this is the most important step in the treatment. For every case in which the infected focus can be treated with surgical drainage or removal of a foreign body, antibiotics are of secondary importance. Table 53.1 shows the results obtained in 77 personally treated patients with Gram-negative rod bacteraemia. The results clearly show that once the treatment of the primary lesion was effective, the prognosis did not differ significantly whether antibiotics were given or not. On the other hand, if the primary focus was not eradicated or if its treatment was ineffective, the prognosis was not significantly changed with the use of selected antibiotics. Nevertheless, in infections in which septic foci are diffuse and not amenable to surgical treatment, e.g., pneumonia, meningitis, or pyelonephritis, antibiotics are the most useful means of therapy.

\section{Supportive therapy}

In patients with impaired immune responses special treatment should be considered. In those with a reduced skin test response two main forms have proved useful: nutritional therapy and an aggressive approach to the infection.

The correction of established malnutrition or the prevention of its occurrence is an effective means of increasing host defence. This appears to be especially important in patients admitted to CCUs, where a high frequency of malnutrition has been shown [17]. Aggressive treatment of infection, especially when a septic focus is capable of being eradicated, is another procedure that has been shown to be effective [11].

In the future, forms of therapy acting directly on abnormalities in the immune response may be used, such as immunostimulation by drugs, infusion of non-specific immunoglobulins, and passive immunotherapy with serum containing antibodies against the toxic part of the LPS. At this time, only limited information is available and such therapy cannot yet be recommended.

\section{References}

1. Abbou C, Cordonnier C, Carlet J, Chopin D, Nebout T, Botto H (1981) Use of computerized tomography (CT) in the diagnosis of renal abscesses (RA). In: Shulman CC (ed) Advances in diagnostic urology. Springer, Berlin Heidelberg New York, pp 185-192

2. Ball AP, Geddes AM (1979) New antibiotics-a review. In: Reeves D and Geddes A (eds) Recent advances in infection, vol. 1. Churchill Livingstone, Edinburgh London New York, p 29

3. Barois A, Bourdain JL (1973) Les septicémies avec hypothermie. In: Goulon M, Rapin M (eds) Réanimation et médecine d'urgence. Expansion, Paris, pp 47-74

4. Barlett VG (1979) Antibiotic-associated pseudomembranous colitis. J Infect Dis 1:530-539

5. Beaufils M, Morel-Maroger L, Sraer JD, Kanfer A, Kourilsky O, Richet G (1976) Acute renal failure of glomerular origin during visceral abscesses. $\mathrm{N}$ Engl J Med 295:185-189

6. Belohradsky BH, Bruchk, Geiss D, Kafetzis D, Marget W, Peters G (1980) Intravenous cefotaxime in children with bacterial meningitis. Lancet I:61-63

7. Berger SA, Nagar H, Weitzman S (1978) Prophylactic antibiotics in surgical procedures. Surg Gynecol Obstet 146: $469-475$

8. Boelld DR, Levitt RG, Melson GL (1979) The role of gallium-67 scintigraphy, ultrasonography and computed tomography in the detection of abdominal abscesses. Semin Nucl Med 9:58-65

9. Chodak GW, Plant ME (1977) Use of systemic antibiotics for prophylaxis in surgery : A critical review. Arch Surg 112:326-334

10. Christou NV, Meakins JL (1979) Neutrophil function in surgical patients: Two inhibitors of granulocyte chemotaxis associated with sepsis. J Surg Res 26:355-363

11. Clumeck N, George C (1981) Immunological aspects of severe bacterial sepsis. Intensive Care Med 7:109-114

12. Cook FV, Farrar WE Jr (1978) Vancomycin revisited. Ann Intern Med 88:813-818

13. Dinarello CA, Wolff SM (1978) Pathogenesis of fever in man. N Engl J Med 298:607-612

14. Dussan J, Regnier B, Darragon T, Teisseire B, Le Gall JR, Lemaire F (1979) Hyperkinetic shock in viral and pneumococcal pneumonias. Intensive Care Med 5:59_ 64

15. Eykyn SJ, Phillips I (1978) Nitronidazole in surgical infections. J Antimicrob Chemother 4:75-81 [Suppl C]

16. Finland $M(1970)$ Changing ecology of bacterial infections as related to antibacterial therapy. J Infect Dis 122: 419-431

17. George C, Carlet J, Sobel A, Intrator L, Robin M, Sabatier C, Rapin M (1980) Circulating immune complexes in patients with Gram-negative septic shock. Intensive Care Med 6:123-127

18. Ingham HR, Sisson PR, Selkon JB (1980) Current concepts of the pathogenetic mechanisms of non sporing anaerobes: Chemotherapeutic implications. J Antimicrob Chemother 6:173-180

19. Kantor FS (1975) Infection, anergy and cell mediated immunity. N Engl J Med 292:629-634 
20. Keighlet MRB, Burdon DW (1979) Antimicrobial prophylaxis in surgery. Pitman Medical, London

21. Kelton JG, Neame PB, Gauldie J, Hirsh J (1979) Elevated platelet associated IgG in the thrombocytopenia of septicemia. N Engl J Med 300:760-734

22. McLean LD, Meakins JL, Raguchi K, Ovignan JP, Omillon KS, Gordon J (1975) Host resistance in sepsis and trauma. Ann Surg 182:207-215

23. Ledingham I, McAedle CS (1978) Prospective study of the treatment of septic shock. Lancet I:1194-1196

24. Le Frock JL, Ellis CA, Weinstein L (1979) The relation between aerobic fecal and oropharyngeal microflora in hospitalized patients. Am J Med Sci 277:275-280

25. Le Gall JR, Mignon FC, Rapin M, Redjem M, Harari A, Bader JP, Soussy CJ (1976) Acute gastroduodenal lesions related to severe sepsis. Surg Gynecol Obstet 142:377380

26. Lhoste F, Lemaire F, Tillement JP, Duval J, Rapin M (1980) Erythromycin lactobionate as a first choice parenteral antibiotic. In: Nelon JD, Grassi C (eds) Severe infective exacerbations of chronic bronchitis. Current chemotherapy and infectious disease. Nineteenth Interscience Conference on Antimicrobial agents and Chemotherapy, vol 2. American Society for Microbiology, Washington DC, pp 1018-1019

27. Mant MJ, King EG (1979) Severe acute disseminated intravascular coagulation. A reappraisal of its pathophysiology clinical significance and therapy based on 47 patients. Am J Med 67:557-563

28. Mouton RP (1980) Choice of an aminoglycoside. J Antimicrob Chemother 6:166-167

29. Nichols PJ (1980) Neurotoxic of Penicillin. J Antimicrob Chemother 6:161-165

30. Rangel DM, Dimbar A, Stewens GH, Cooper R, Fonkalsrud EW (1977) The hepatic response to endotoxin shock: Hemodynamic and enzymatic observations. Intensive Care Med 3:47-53
31. Rapin M, Duval J, Le Gall JR, Soussy CJ, Lemaire F, Harari A (1975) Les septicémies de surinfection en réanimation. Leur prévention par la restriction de $l^{\prime}$ antibiothérapie. Nouv Presse Med 4:483-486

32. Sagel SS, Staaley RJ, Levitt RG, Geisse (1977) Computed tomography of the kidney. Radiology 124:359-370

33. Schiffman DO (1975) Evaluation of an anti-infective combination. Trimethoprim-Sulfamethoxazole. JAMA 231:635-637

34. Semel JD, Trenholme GM, Harris AA, Jupa JE, Levin S (1978) Pseudomonas maltophilia pseudosepticemia. Am J Med 64:403-406

35. Shattil SJ, Bennett JS, McDonough M, Turnbull J (1980) Carbenicillin and penicillin $\mathrm{G}$ inhibit platelet function in vitro by impairing the interaction of agonists with the platelet surface. J Clin Invest 65:329-337

36. Siegel JM, Cerra FB, Coleman B, Giovannini I, Shetye M, Border JR, McMenamy RH (1979) Physiological and metabolic correlations in human sepsis. Surgery 86:163193

37. Speller DCE (1980) Hospital infection by multiresistant Gram-negative bacilli. J Antimicrob Chemother 6:19701972

38. Stoddart JC (1974) Gram-negative infections in the ICU. Crit Care Med 2:17-22

39. Stone HH, Haney BB, Kolb LD, Geheber GE, Hooper CA (1979) Prophylactic and preventive antibiotic therapy-Timing, duration and economics. Ann Surg 189:691-699

40. Weinstein RJ, Young LS (1976) Neutrophil function in Gram negative rod bacteriema. J Clin Invest 58:190-198

41. Wiles JB, Cerra FB, Siegel JM, Border JR (1980) The systemic septic response: Does the organism matter? Crit Care Med 8:55-60 